PROCEEDINGS OF THE

AMERICAN MATHEMATICAL SOCIETY

Volume 135, Number 3, March 2007, Pages 731-741

S 0002-9939(06)08560-1

Article electronically published on August 31, 2006

\title{
LAX PAIRS FOR HIGHER-DIMENSIONAL EVOLUTION PDES AND A 3+1 DIMENSIONAL INTEGRABLE GENERALIZATION OF THE BURGERS EQUATION
}

\author{
M. RUDNEV, A. V. YUROV, AND V. A. YUROV
}

(Communicated by Mark J. Ablowitz)

\begin{abstract}
We construct Lax pairs for general $d+1$ dimensional evolution equations in the form $u_{t}=F[u]$, where $F[u]$ depends on the field $u$ and its space derivatives. As an example we study a $3+1$ dimensional integrable generalization of the Burgers equation. We develop a procedure to generate some exact solutions of this equation, based on a class of discrete symmetries of the Darboux transformation type. In the one-dimensional limit, these symmetries reduce to the Cole-Hopf substitution for the Burgers equation. It is discussed how the technique can be used to construct exact solutions for higher-dimensional evolution PDEs in a broader context.
\end{abstract}

\section{INTRODUCTION}

Most of the known completely integrable nonlinear evolution PDEs are $1+1$ dimensional [1, 12. In space dimension $d \geq 2$, the existing theory encounters fundamental algebraic and geometric obstructions. Integrability of nonlinear PDEs is closely related (yet certainly not equivalent) to the existence of $[L, A]$, or Lax pairs. It turns out that for any $d \geq 1$ and any nonlinear scalar evolution equation

$$
u_{t}=F[u],
$$

where $F[u]$ is a finite algebraic expression containing $u$ and its space derivatives, there exists an $[L, A]$ pair. Let us illustrate it for $d=1,2,3$.

(1) If $d=1$, the $[L, A]$ pair for equation (1.1) has the following form:

$$
\begin{aligned}
& \psi_{x}=u_{x} \psi, \\
& \psi_{t}=a_{11} \psi_{x x}+\left(a-2 a_{11}\right) \psi_{x}+\left(F[u]+a_{11}\left(u_{x}^{2}-u_{x x}\right)-a u_{x}\right) \psi,
\end{aligned}
$$

where $a_{11}, a$ are arbitrary constants and $u=u(x, t), \psi=\psi(x, t)$.

Received by the editors November 29, 2004 and, in revised form, May 16, 2005 and September 23, 2005.

2000 Mathematics Subject Classification. Primary 35Q53, 35Q58.

Key words and phrases. Integrable evolution equations, Lax pairs, Darboux transformation, Burgers equation.

(C)2006 American Mathematical Society Reverts to public domain 28 years from publication 
(2) If $d=2$, we have

$$
\begin{aligned}
\psi_{x y}= & u_{y} \psi_{x}+u_{x} \psi_{y}+\left(u_{x y}-u_{x} u_{y}\right) \psi, \\
\psi_{t}= & a_{11} \psi_{x x}+a_{22} \psi_{y y}+\left(a-2 a_{11} u_{x}\right) \psi_{x}+\left(b-2 a_{22} u_{y}\right) \psi_{y} \\
& +\left(F[u]+a_{11}\left(u_{x}^{2}-u_{x x}\right)+a_{22}\left(u_{y}^{2}-u_{y y}\right)-a u_{x}-b u_{y}\right) \psi,
\end{aligned}
$$

where $a_{11}, a_{22}, a, b$ are arbitrary constants and $u=u(x, y, t), \psi=\psi(x, y, t)$.

(3) If $d=3$, the analog of (1.2 1.3) is

$$
\begin{aligned}
\psi_{x y z}= & u_{y} \psi_{x z}+u_{x} \psi_{y z}+u_{z} \psi_{x y} \\
& +\left(u_{x y}-u_{x} u_{y}\right) \psi_{z}+\left(u_{y z}-u_{z} u_{y}\right) \psi_{x}+\left(u_{x z}-u_{x} u_{z}\right) \psi_{y} \\
& +\left(u_{x y z}-u_{x} u_{y z}-u_{y} u_{x z}-u_{z} u_{x y}+u_{x} u_{y} u_{z}\right) \psi, \\
\psi_{t}=a_{11} & \psi_{x x}+a_{22} \psi_{y y}+a_{33} \psi_{z z}+2\left(a_{12} \psi_{x y}+a_{13} \psi_{x z}+a_{23} \psi_{y z}\right) \\
& +\left(a-2\left(a_{11} u_{x}+a_{12} u_{y}+a_{13} u_{z}\right)\right) \psi_{x}+\left(b-2\left(a_{12} u_{x}+a_{22} u_{y}+a_{23} u_{z}\right)\right) \psi_{y} \\
& +\left(c-2\left(a_{13} u_{x}+a_{23} u_{y}+a_{33} u_{z}\right)\right) \psi_{z}+\left(F[u]+a_{11}\left(u_{x}^{2}-u_{x x}\right)\right. \\
& +a_{22}\left(u_{y}^{2}-u_{y y}\right)+a_{33}\left(u_{z}^{2}-u_{z z}\right)+2\left(a_{12}\left(u_{x} u_{y}-u_{x y}\right)\right. \\
& \left.\left.+a_{13}\left(u_{x} u_{z}-u_{x z}\right)+a_{23}\left(u_{y} u_{z}-u_{y z}\right)\right)-a u_{x}-b u_{y}-c u_{z}\right) \psi,
\end{aligned}
$$

where $a_{m n}(m, n=1, \ldots, 3), a, b$ are arbitrary constants and $u=u(x, y, z, t)$, $\psi=\psi(x, y, z, t)$.

For the general $d \geq 1$, the first equation, or the $L$-equation, of the pair will have order $d$, while the second equation, or the $A$-equation, will always be of the second order in the space variables.

Clearly, the existence of the $[L, A]$ pair does not in general guarantee integrability of the corresponding PDE. However in many cases (this issue is addressed in the final section of this note in some detail) the pair may still enable one to construct nontrivial exact solutions of the equation in question.

Until the last section, let us study the special case when $F[u]$ is such that the coefficient multiplying $\psi$ in the $A$-equation is zero. If $d=1$, this yields the Burgers equation, whose Lax pair is

$$
\psi_{x}=u_{x} \psi, \quad \psi_{t}=a \psi_{x x}+b \psi_{x},
$$

with the compatibility condition

$$
w_{t}=a\left(2 w w_{x}+w_{x x}\right)+b w_{x},
$$

where $w=u_{x}$.

Recall that the standard form of the Burgers equation for $U=U(x, t)$ is

$$
U_{t}+U U_{x}-\nu U_{x x}=0,
$$

and it can be fully linearized via the Cole-Hopf substitution ([6], 9])

$$
U=-2 \nu \partial_{x} \log \Theta,
$$

which transforms it to the heat equation (with diffusivity $\nu$ ) for the quantity $\Theta(x, t)$.

Despite its being linearizable, the Burgers equation is closely related to bona fide nonlinear PDE hierarchies, such as $\mathrm{KdV}$ and $\mathrm{mKdV}$, 8]. It plays an important rôle in physical models characterized by the balance between nonlinearity and dissipation, 3] (rather than dispersion, which makes the Burgers equation quite special 
among other evolution equations). For some physical models involving the Burgers equation see e.g. [5], 4], [13].

If $d>1$, the family (1.1) of evolution equations provides a wide generalization of the Burgers equation. Each member of the family allows for a Lax pair and can occasionally be linearized. If this is the case, one can spot certain displays of linearizability in the equation's symmetry structure, as it happens for instance with the Thomas equation; see 14.

We shall further deal with $d=3$. In the recent work [15, the $2+1$ dimensional BLP (Boiti, Leon, and Pempinelli) system was studied in the same vein, namely as an integrable two-dimensional generalization of the Burgers equation.

Henceforth, consider the following $3+1$ dimensional representative of the family (1.1):

$$
\begin{aligned}
K[u] \equiv & u_{t}+a_{1}\left(u_{x}^{2}-u_{x x}\right)+a_{2}\left(u_{z}^{2}-u_{z z}\right)+b_{1}\left(u_{x} u_{y}-u_{x y}\right) \\
& \quad+b_{2}\left(u_{x} u_{z}-u_{x z}\right)-\rho u_{x}-\mu u_{y}-\lambda u_{z} \\
=0 &
\end{aligned}
$$

where $u=u(x, y, z, t)$ and the rest of the parameters are constants. Let us repeat that this equation results from the $[L, A]$ pair (1.4) in a special case, when $F[u]$ is such that it zeroes the last bracket in the $A$-equation of the system. The general situation is dealt with in Section 5 .

Equation (1.8) contains dissipative terms and has the following properties:

(1) It is completely integrable.

(2) In the $1+1$ dimensional limit it gives the Burgers equation.

(3) It admits a class of discrete symmetries of the Darboux transformation type. Namely, to take advantage of these symmetries, one has to solve the Lax pair equations. This enables one to construct a rich family of the exact solutions of the equation. In particular, it becomes possible to fulfill a $3+1$ dimensional dressing of the Burgers equation.

(4) In the $1+1$ dimensional limit, the symmetries in question reduce to the Cole-Hopf transformation.

Equation (1.8) is completely integrable, as it allows for complete linearization, after multiplying it by $\exp (-u)$ and introducing the new variable $v=\exp (-u)$. In this sense, it represents a very close analog of the Burgers equation. It may appear that the fact of linearizability makes the development of "nonlinear" methods for this equation superfluous. There are several principal objections against that.

The first and the main one is the fact that equation (1.8) represents in a sense a limit case of the class of nonlinear equations, resulting from the $[L, A]$ pair (1.4). In the general case, point linearization would be impossible. However, the technique developed below is based solely on the intertwining properties of the $L$-operator and to some extent (see Section 5) applies to the general case. In the same vein, the Burgers equation, although linearizable, has its proper niche among nonlinear hierarchies; see 8 ] and the references contained therein.

The dressing procedure developed further does not contain a spectral parameter. Thus, from the point of view of Inverse Scattering the above Lax pair is not likely to be useful. This is hardly an exception however in the case $d>1$. Rather, the situation is similar to the case of $2+1$ integrable models, such as for instance the Davey-Stewartson equations. (See e.g. [10].) In general, it is not fully understood to what extent the presence of the spectral parameter (and Inverse Scattering) should 
be characteristic of multi-dimensional models, or it is the special feature of the case $d=1$ (although in the case $d=2$, the spectral parameter can be introduced and the spectral theory worked out). For recent results dealing with $2+1$ dimensional generalizations of spectral theory methods, see [2] and the references contained therein.

At last, the technique can be applied in the "inverse" direction. The linearizing substitution results in strictly positive real solutions of the corresponding linear equation. Hence, one can use the exact solutions of the nonlinear equation (1.8) in order to construct positive solutions of the underlying linear equation, which may be of some physical sense.

\section{Statement of Results}

In the $1+1$ dimensional limit, equation (1.8) reduces to the dissipative Burgers equation. Indeed, imposing a one-dimensional reduction $\partial_{y}=\partial_{z}=0$, and defining the quantity

$$
\xi(x, t)=u_{x}(x, t)
$$

we obtain

$$
\xi_{t}-\rho \xi_{x}-a_{1} \xi_{x x}+2 a_{1} \xi \xi_{x}=0 .
$$

The latter equation becomes the Burgers equation after either changing $t \rightarrow t^{\prime}$, such that

$$
\partial_{t^{\prime}}=\partial_{t}-\rho \partial_{x}
$$

or directly letting $\rho=0$. Clearly, the reduction $\partial_{x}=\partial_{y}=0$ has a similar effect, while the reduction $u=u(y, t)$ yields

$$
u_{t}-\mu u_{y}=0
$$

a linear equation. In view of the above, equation (1.8) can be viewed as a special nonisotropic three-dimensional extension of the Burgers equation. To emphasize this, let $w=u_{x}$ and consider $\mu=\rho=\lambda=0$. Rewrite (1.8) as follows:

$$
\begin{gathered}
w_{t}+2 a_{1} w w_{x}+b_{1} w w_{y}+b_{2} w w_{z}-a_{1} w_{x x}-a_{2} w_{z z}-b_{1} w_{x y}-b_{2} w_{x z} \\
+b_{1} u_{y} w_{x}+b_{2} u_{z} w_{x}+2 a_{2} u_{z} w_{z}=0 .
\end{gathered}
$$

One can see that in the "physical" realization of equation (2.3) one should have $a_{1}, a_{2}>0$, for otherwise the problem is ill-posed. Further in this note we construct some exact solutions of equation (2.3); see Examples 4.1 and 4.3. We observe however, that in the apparently "non-physical" case $a_{1} a_{2}<0$, the latter equation admits three-dimensional stationary localized solutions, which may or may not possess physical meaning; see Example 4.2

Our main result is the following theorem.

Theorem 2.1. Let $u(x, y, z, t)$ be a particular solution of equation (1.8) and let $\psi=\psi(x, y, z, t)$ satisfy the following linear equation:

$$
\begin{gathered}
\psi_{t}=a_{1} \psi_{x x}+a_{2} \psi_{z z}+b_{1} \psi_{x y}+b_{2} \psi_{x z}+\left(\rho-2 a_{1} u_{x}-b_{2} u_{z}-b_{1} u_{y}\right) \psi_{x} \\
+\left(\mu-b_{1} u_{x}\right) \psi_{y}+\left(\lambda-2 a_{2} u_{z}-b_{2} u_{x}\right) \psi_{z} \equiv \boldsymbol{A}[u] \psi
\end{gathered}
$$

Then any $\tilde{u}_{k l m}=\tilde{u}_{k l m}(x, y, z, t)$, defined as

$$
\tilde{u}_{k l m}=u-\log \left(\left(\partial_{x}-u_{x}\right)^{k}\left(\partial_{y}-u_{y}\right)^{l}\left(\partial_{z}-u_{z}\right)^{m} \psi\right),
$$

for some $(k, l, m) \in \mathbb{Z}_{+}^{3}$, is also a solution of equation (1.8). 
Above, $\mathbb{Z}_{+}$denotes nonnegative integers; $\mathbb{Z}_{++}$further stands for positive integers.

Theorem 2.1 is based on a Lax pair, which is a particular case of (1.4), where some parameters have been assigned zero values.

Proposition 2.2. Equation (1.8) admits a Lax pair, with the A-equation $\psi_{t}=$ $\boldsymbol{A}(u) \psi($ see (2.4)) and the L-equation

$$
\begin{aligned}
& \psi_{x y z}=u_{z} \psi_{x y}+u_{y} \psi_{x z}+u_{x} \psi_{y z}+\left(u_{y z}-u_{y} u_{z}\right) \psi_{x}+\left(u_{x z}-u_{x} u_{z}\right) \psi_{y} \\
& +\left(u_{x y}-u_{x} u_{y}\right) \psi_{z}+\left(u_{x y z}-u_{y z} u_{x}-u_{y x} u_{z}-u_{x z} u_{y}+u_{x} u_{y} u_{z}\right) \psi
\end{aligned}
$$

Observe that the spectral equation (2.6) of the Lax pair can be rewritten as follows:

$$
\left(\partial_{x}-u_{x}\right)\left(\partial_{y}-u_{y}\right)\left(\partial_{z}-u_{z}\right) \psi \equiv \boldsymbol{L}_{1}[u] \boldsymbol{L}_{2}[u] \boldsymbol{L}_{3}[u] \psi \equiv \boldsymbol{L}[u] \psi=0 .
$$

Also observe that if we introduce the operator $\boldsymbol{A}^{\prime}[u]=\boldsymbol{A}[u]+K[u]$, the compatibility condition of the Lax pair equations (2.6) and (2.4) will be reduced to the identity. Namely the operators $\boldsymbol{L}[u]$ and $\boldsymbol{B}^{\prime}[u]=\partial_{t}-\boldsymbol{A}^{\prime}[u]$ commute: $\left[\boldsymbol{L}[u], \boldsymbol{B}^{\prime}[u]\right]=0$.

Successive iteration of (2.5) results in the following corollary.

Corollary 2.3. Let $\left\{\psi_{i}\right\}, i=1, \ldots, N$, be a set of particular solutions of the $A$ equation (2.4). Given a potential u satisfying equation (1.8), new solutions of this equation can be generated as follows:

$$
\tilde{u}=u-\log \left(\prod_{i=1}^{N} \boldsymbol{L}_{1}^{k_{i}}[u] \boldsymbol{L}_{2}^{l_{i}}[u] \boldsymbol{L}_{3}^{m_{i}}[u] \psi_{i}\right),
$$

where $\left(k_{i}, l_{i}, m_{i}\right) \in \mathbb{Z}_{+}^{3}$.

Remark 2.4. Note that in the formulation of Theorem 2.1 there is no mention of the $L$-equation of the pair. It is the special choice of the quantity $F[u]$ in equation (1.8) that makes the $L$-equation play only an auxiliary rôle to determine the intertwining properties of the operators used to prove the theorem; cf. (3.2). In particular, the $L$-equation in general does not have to be satisfied by the function $\psi$ in Theorem 2.1. This happens in particular in Example 4.3 in the sequel, while in Examples 4.1, 4.2 the $L$-equation is satisfied. If the $L$-equation is satisfied, then one should additionally require that $(k, l, m) \notin \mathbb{Z}_{++}^{3}$ in Theorem 2.1 and the following Corollary 2.3. as (2.6) states that $\boldsymbol{L}=\boldsymbol{L}_{1} \boldsymbol{L}_{2} \boldsymbol{L}_{3}$ is an annihilator of $\psi$. Generally speaking, the formulae (2.5), (2.8) should be viewed as computation rules, rather than be characteristic of some function space. In particular, the choice $\left(k_{i}, l_{i}, m_{i}\right)$ not in $\mathbb{Z}_{++}^{3}$ alone cannot ensure that the quantity $\tilde{u}$ be generally nonsingular.

Earlier it was shown that the Burgers equation (2.2) follows from equation (1.8) after letting $\partial_{y}=\partial_{z}=0$. Equivalently, if we set $u=u(x, t)$ in (2.5) and use it with $u \equiv 0, k=1$, then after differentiation with respect to $x$, the expression (2.5) results in the Cole-Hopf substitution.

\section{Proof of Theorem 2.1}

The proof of the theorem is based on the following observation.

Lemma 3.1. Let $\psi$ be a solution of the Lax pair equations (2.6), (2.4) with the potential u, which is a solution of equation (1.8). Then the function

$$
\tilde{\psi}_{k l m}=\boldsymbol{L}_{1}^{k}[u] \boldsymbol{L}_{2}^{l}[u] \boldsymbol{L}_{3}^{m}[u] \psi, \quad(k, l, m) \in \mathbb{Z}_{+}^{3},
$$


also satisfies the $[L, A]$ equations (2.6), (2.4), with the same potential $u$.

If $\psi$ is a solution of the A-equation (2.4) alone, then $\tilde{\psi}_{k l m}$ computed by (3.1) is also a solution of (2.4).

The lemma follows by induction from the following commutation relations, whose verification is a direct computation: for $i, j=1,2,3$ one has

$$
\left[\boldsymbol{L}_{i}[u], \boldsymbol{L}[u]\right]=\left[\boldsymbol{L}_{i}[u], \boldsymbol{B}[u]\right]=\left[\boldsymbol{L}_{i}[u], \boldsymbol{L}_{j}[u]\right]=0,
$$

where $\boldsymbol{B}[u]=\partial_{t}-\boldsymbol{A}[u]$.

To prove Theorem 2.1, let us introduce three intertwining operators

$$
\boldsymbol{D}_{i}=f_{i} \partial_{i}-g_{i}, \quad i=1,2,3,
$$

with the quantities $f_{i}, g_{i}$ to be determined (notation-wise, above $\partial_{1,2,3}=\partial_{x, y, z}$, respectively), such that the operators $\boldsymbol{D}_{i}$ have the following property: for some $u_{i}=u_{i}(x, y, z, t)$,

$$
\boldsymbol{L}\left(u_{i}\right) \boldsymbol{D}_{i}=\boldsymbol{D}_{i} \boldsymbol{L}[u], \quad \boldsymbol{B}\left(u_{i}\right) \boldsymbol{D}_{i}=\boldsymbol{D}_{i} \boldsymbol{B}[u] .
$$

The commutation relations (3.3) determine the maps $u \rightarrow u_{i}$, which result from the substitution of (3.2) into (3.3). The explicit form of the operators $\boldsymbol{D}_{i}$ can be found as follows.

Substituting (3.2) into (3.3) and equating the components at the same partial derivatives results in a system of nonlinear equations (the reader is spared the latter system that looks rather cumbersome), whence it follows that

$$
\boldsymbol{D}_{i}=\mathrm{e}^{-v}\left(\boldsymbol{L}_{i}[u]-c_{i}\right), \quad u_{i}=\tilde{u}=u-v,
$$

where $c_{i}$ are constants and will be further assigned zero values. If $u$ is a solution of (1.8), then $\tilde{u}$ is a new solution of (1.8), provided that $v=v(x, y, z, t)$ satisfies the following nonlinear equation:

$$
\begin{aligned}
v_{t}= & a_{1}\left(v_{x x}+v_{x}^{2}\right)+a_{2}\left(v_{z z}+v_{z}^{2}\right)+b_{1}\left(v_{x y}+v_{x} v_{y}\right)+b_{2}\left(v_{x z}+v_{x} v_{z}\right) \\
& +\left(\rho-2 a_{1} u_{x}-b_{2} u_{z}-b_{1} u_{y}\right) v_{x}+\left(\mu-b_{1} u_{x}\right) v_{y}+\left(\lambda-2 a_{2} u_{z}-b_{2} u_{x}\right) v_{z} .
\end{aligned}
$$

Therefore the relations (3.4) or explicitly (3.5) indicate that for $u \equiv 0$, the function " $-v$ " satisfies equation (1.8).

Let us consider the quantity $\psi=\mathrm{e}^{u}$ and substitute it into (2.4). If $u$ is a solution of (1.8), then $\psi=\mathrm{e}^{u}$ will satisfy (2.4). On the other hand, by Lemma3.1)(namely its last statement), the quantities $\tilde{\psi}_{k l m}$ defined by the relation (3.1) are also solutions of (2.4), with the same potential $u$. Rewriting them as $\tilde{\psi}_{k l m}=\exp \left(v_{k l m}\right)$ and substituting into (2.4), one verifies that the quantities $v_{k l m}$ indeed satisfy equation (3.5). Theorem 2.1 and the formula (2.5) now follow from the second relation from (3.4).

Observe that the $L$-equation has played but an auxiliary rôle throughout the proof. I.e., if $\psi$ solves the $A$-equation, we can formally let $u=\log \psi$, and then $\left(\partial_{x}-u_{x}\right) \psi=0$ is just a truism.

Remark 3.2. The formula (2.5) has a countenance similar to the Darboux transformation, which is a standard tool for constructing exact solutions of nonlinear PDEs (usually in $1+1$, more rarely in $2+1$ dimensions) which admit Lax pairs. See e.g. 11] for general theory, applications and references. Strictly speaking however, the formula (2.5) does not represent a bona fide Darboux transform for the following reasons. 
(1) The Darboux transformation, representing discrete symmetries of a particular Lax pair, possesses a nontrivial kernel on the solution space of the pair. This is precisely the property that enables one to write down the Crum determinant formulae (see [7]) for successive Darboux transforms. However, the transformation (2.5) does not have such a property.

(2) In the Darboux transform, one always needs the solution of the full $[L, A]$ pair. Contrary to this, in the proof of Theorem 2.1, in order to get (2.5), we have used the solution of the $A$-equation (2.4) only. To this effect, the transformation (2.5) is similar to the Cole-Hopf transformation and points towards the possibility of linearization of equation (1.8).

\section{Some ExaCt solutions}

Let us use Theorem 2.1 to construct some exact solutions of equation (2.3) (which is the equation for $u_{x}$, where $u$ is a solution of equation (1.8) with $\mu=\lambda=\rho=0$ ). We consider equation (2.3), rather than (1.8), as it bears a closer resemblance to the Burgers equation and is more likely to be interesting from the physical point of view. Observe that in the first two examples, both Lax pair equations (with the background potential $u$ ) will be satisfied by the function $\psi$, while in the third example, it is only the $A$-equation; cf. Remark 2.4 .

Example 4.1. Consider dressing on the vacuum background $u \equiv 0$. One solution of the Lax pair equations (2.6), (2.4) is

$$
\begin{gathered}
\psi(x, y, z, t)=c_{1} \mathrm{e}^{\alpha\left(\alpha a_{1}+\beta b_{1}\right) t} \cosh (\alpha x+\beta y)+c_{2} \mathrm{e}^{\left(a_{1} a^{2}+a_{2} b^{2}+a b b_{2}\right) t} \cosh (a x+b z) \\
+c_{3} \mathrm{e}^{a_{2} c^{2} t} \cosh (c z),
\end{gathered}
$$

where $\alpha, \beta, a, a_{1}, a_{2}, b, b_{1}, b_{2}, c_{1}, c_{2}, c_{3}$ are some real constants. Let us choose them such that

$$
\beta=-\frac{\alpha a_{1}}{b_{1}}, \quad b=-\frac{a}{2 a_{2}}\left(b_{2} \pm \sqrt{b_{2}^{2}-4 a_{1} a_{2}}\right) .
$$

Substituting now (4.1) into (2.5), we compute the quantity $\tilde{u}_{k l m}$. Further differentiating it with respect to $x$ and choosing $k=l=m=0$, we obtain a solution $w$ to equation (2.3) as follows:

$$
w(x, y, z, t)=-\frac{\alpha c_{1} \sinh (\alpha x+\beta y)+a c_{2} \sinh (a x+b z)}{c_{1} \cosh (\alpha x+\beta y)+c_{2} \cosh (a x+b z)+c_{3} \mathrm{e}^{a_{2} c^{2} t} \cosh (c z)} .
$$

Example 4.2. Let us consider once again $u \equiv 0$. As a solution of the Lax pair equations now take

$$
\psi(x, y, z, t)=a^{2} x^{2}+b^{2} y^{2}+c^{2} z^{2}+2\left(a_{1} a^{2}+a_{2} c^{2}\right) t+s^{2},
$$

where $a, a_{1}, a_{2}, b, c, s$ are some real constants. In the same vein as in the previous example, we obtain

$$
w(x, y, z, t)=-\frac{2 a^{2} x}{a^{2} x^{2}+b^{2} y^{2}+c^{2} z^{2}+2\left(a_{1} a^{2}+a_{2} c^{2}\right) t+s^{2}},
$$

an exact solution of equation (2.3). Does this solution have a physical meaning? Apparently it describes a rationally localized impulse, vanishing as $t \rightarrow+\infty$. To ensure that it is nonsingular for $t \geq 0$, one should impose the inequality $a_{2} \geq$ 
$-a^{2} a_{1} / c^{2}$ on the coefficients. In the case $a_{2}=-a^{2} a_{1} / c^{2}$, the solution becomes stationary.

It was noted earlier however that if $a_{1} a_{2}<0$, the problem described by equation (2.3) is ill-posed. On the other hand, this is precisely the case when the solution (4.5) is stationary. The fact of the existence of a localized stationary solution in an equation containing dissipative terms may appear a physical nonsense. One can see that the constants $a_{1}$ and $a_{2}$ come up in the dissipative terms of equation (2.3). The constant $a_{1}$ characterizes the dissipation along the $x$-axis and $a_{2}$ along the $z$ axis. The case when $a_{1}$ and $a_{2}$ have different signs suggests that dissipation in one direction is compensated by instability in the other. These two effects balance each other through nonlinearity, resulting in the appearance of the stationary solution (4.5). This solution can be regarded as a three-dimensional dissipative structure. A similar scenario occurs for two-dimensional stationary solutions of the BLP equation, described in [15. It is arguable whether or not there is a physical meaning to such phenomena.

Example 4.3. Let us now illustrate how some exact solutions of the three-dimensional equation (2.3) can be constructed on the basis of the solutions of the $1+1$ dimensional Burgers equation. Consider equation (2.2) for the unknown $\xi(x, t)$. Suppose $\lambda=\mu=\rho=0$ and let $a_{1}=\nu$ in (2.2). One can see that the quantity $U(x, t)=2 \nu \xi(x, t)$ satisfies the one-dimensional Burgers equation (1.6).

As a starting point let us take a shock wave solution of the Burgers equation, e.g.

$$
\xi=u_{x}=\frac{v-\nu a}{2 \nu}+\frac{a}{1+\mathrm{e}^{a(x-v t)}},
$$

where $a$ and $v$ are constants. Let us seek a solution of (2.4) in the superposition form

$$
\psi=\sum_{k=1}^{N} A_{k}(\eta) \mathrm{e}^{\beta_{k} y+\gamma_{k} z},
$$

where $\eta=x-v t$, and the $2 N$ quantities $\beta_{k}$ and $\gamma_{k}$ can in general be functions of $\eta$. For simplicity however let us treat them as constants to be determined.

Substituting (4.7) into (2.4) yields $N$ linear equations for the unknowns $A_{k}(\eta)$ :

$$
\nu \ddot{A}+(v+\sigma-2 \nu \xi) \dot{A}+\left(a_{2} \gamma^{2}-\sigma \xi\right) A=0 .
$$

In equation (4.8) above, the subscripts ${ }_{k}$ for the quantities $A_{k}, \beta_{k}, \gamma_{k}$ and $\sigma_{k} \equiv$ $b_{1} \beta_{k}+b_{2} \gamma_{k}$ have been omitted, the quantity $\xi$ has come from (4.6), and the dot denotes differentiation with respect to $\eta$.

The expression (4.8) can be simplified further. With the notation

$$
q=\xi(x, t)-\xi_{0}, \quad \mathrm{e}^{a \eta}=\frac{a}{q}-1, \quad \xi_{0}=\frac{v-\nu a}{2 \nu},
$$

equation (4.8), rewritten in terms of $q$, becomes

$$
\nu\left(q^{2}-a q\right)^{2} A^{\prime \prime}(q)+\sigma\left(q^{2}-a q\right) A^{\prime}(q)+(\delta-\sigma q) A(q)=0,
$$

where $\delta=a_{2} \gamma^{2}-\sigma \xi_{0}$, and the prime denotes differentiation with respect to $q$. The latter equation can be further simplified by substituting

$$
A(q)=W(q)\left(\frac{q}{q-a}\right)^{\sigma /(2 \nu a)} .
$$


This reduces (4.9) to the following equation for the quantity $W(q)$ :

$$
\frac{W^{\prime \prime}(q)}{W(q)}=\frac{2 a \sigma \nu-4 \delta \nu+\sigma^{2}}{4 \nu^{2}(q-a)^{2} q^{2}} .
$$

In a particular case, when the right-hand side in (4.11) is zero, one can solve the $A$-equation (2.4) of the $[L, A]$ pair explicitly. Bringing the subscripts ${ }_{k}$ back into the notation, this is tantamount to choosing

$$
\beta_{k}=\frac{-b_{2} \gamma_{k}-v \pm \sqrt{v^{2}+4 a_{2} \nu \gamma_{k}^{2}}}{b_{1}}
$$

in (4.7). Then

$$
\psi=\sum_{k=1}^{N}\left(W_{k} q+V_{k}\right)\left(\frac{q}{q-a}\right)^{\sigma_{k} /(2 \nu a)} \mathrm{e}^{\beta_{k} y+\gamma_{k} z},
$$

where $W_{k}$ and $V_{k}$ are arbitrary constants. Substituting (4.12) into (2.5), we obtain a family of exact solutions of equations (1.8) or (2.3). The described procedure can be regarded physically as a three-dimensional dressing of one-dimensional shock waves.

\section{THE General CASE}

Let us now address the general case of $F[u]$, when the linear in $\psi$ term in the $A$-equation of the pairs (1.2)-(1.4) is nonzero. The situation now is certainly more complicated than has been discussed so far. However, it turns out that the above-described Darboux-transformation-like procedure can be used to establish the Bäcklund transformations between different evolution equations, hence enabling one to construct some exact solutions of these equations.

We shall further outline a general procedure, which we expect to address in more detail in a separate paper. To reduce the calculation volume, let us further confine ourselves to the first spatially nontrivial dimension $d=2$.

Hence, let us consider the general equation (1.1), where the quantity $F[u]$ depends on $u(t, x, y)$ as well as its spatial derivatives: $F[u]=F\left(u, u_{x}, u_{y}, u_{x x}, u_{x y}, \ldots\right)$. One of the Darboux transforms for this equation remains the same:

$$
\psi \rightarrow \tilde{\psi}=\mathrm{e}^{\tilde{u}-u}\left(\psi_{x}-u_{x} \psi\right) .
$$

The new quantity $\tilde{u}(t, x, y)$ satisfies a new nonlinear equation as follows:

$$
\tilde{u}_{t}=\tilde{F}[\tilde{u}],
$$

where the quantity $\tilde{F}[\tilde{u}]=\tilde{F}\left(\tilde{u}, \tilde{u}_{x}, \tilde{u}_{y}, \tilde{u}_{x x}, \tilde{u}_{x y}, \ldots\right)$ remains arbitrary. In fact, we can choose the expressions $F$ and $\tilde{F}$ in a convenient way. Take for instance

$$
F[u]=u_{x x}+u_{y y}+u, \quad \tilde{F}[\tilde{u}]=\tilde{u}_{x x}+\tilde{u}_{y y}+u^{3},
$$

etc. The choice of the expressions for $F$ and $\tilde{F}$ should be constrained by the following condition: both equations (1.1) and (5.1) must allow for at least two integrals of motion in the form

$$
C_{1}\left(u, u_{x}, u_{y}\right)=E_{1}, \quad C_{2}\left(u, u_{x}, u_{y}\right)=E_{2},
$$

for (1.1) and

$$
\tilde{C}_{1}\left(\tilde{u}, \tilde{u}_{x}, \tilde{u}_{y}\right)=\tilde{E}_{1}, \quad \tilde{C}_{2}\left(\tilde{u}, \tilde{u}_{x}, \tilde{u}_{y}\right)=\tilde{E}_{2},
$$


for (5.1). Using (5.2), one can express the space derivatives $u_{x}, u_{y}$ via $u$ :

$$
u_{x}=\xi_{1}\left(E_{1}, E_{2}, u\right)=A_{1}(u), \quad u_{y}=\xi_{2}\left(E_{1}, E_{2}, u\right)=A_{2}(u) .
$$

This clearly enables one to get rid of the rest of the derivatives as well, by expressing for instance

$$
\begin{aligned}
& u_{x x}=\frac{d A_{1}(u)}{d u} u_{x}=A_{1}^{\prime}(u) A_{1}(u)=B_{1}(u), \\
& u_{x y}=\frac{d A_{1}(u)}{d u} u_{y}=A_{1}^{\prime}(u) A_{2}(u)=B_{2}(u),
\end{aligned}
$$

etc. Hence, the quantity $F[u]=F\left(u, u_{x}, u_{y}, u_{x x}, u_{x y}, \ldots\right)=G(u)$ can be rewritten as a function of $u$ only. In the same fashion, one can use (5.3) to rewrite $\tilde{F}$ as a function of $\tilde{u}$ only: $\tilde{F}[u]=\tilde{G}(\tilde{u})$.

Now consider the functional dependence

$$
\tilde{u}=f(u) \text {, }
$$

to be determined. The function $f$ gets fully determined by the quantities $F$ and $\tilde{F}$ above. Let us show that if $F$ and $\tilde{F}$ satisfy the conditions (5.2) and (5.3), then the dependence (5.4) can be found in quadratures. Substituting (5.4) into (5.1) we get

$$
\tilde{u}_{t}=\frac{d f(u)}{d u} u_{t}=f^{\prime}(u) G(u)=\tilde{G}(f),
$$

and hence

$$
\int \frac{d f}{\tilde{G}(f)}=\int \frac{d u}{G(u)} .
$$

Integration (whenever feasible) determines (explicitly or implicitly) the functional dependence (5.4), which represents the Bäcklund transformation between equations (1.1) and (5.1).

The above-described procedure can be applied to the case $d=3$, allowing for the pair (1.4). Similar to the case $d=2$, the main issue is to choose the quantities $F$ and $\tilde{F}$ to ensure that there exists a sufficient number of first integrals that would enable elimination of the space derivatives of $u$ and $\tilde{u}$ in $F$ and $\tilde{F}$.

In conclusion, let us remark that it appears indeed valuable if one could exhibit a nontrivial example of a $2+1$ dimensional physically relevant equation for which the above-outlined technique can be effectively carried out. At this stage in particular, it is not clear whether looking for auto-Bäcklund transformations would essentially facilitate this task. We intend to address this and other issues that may eventually enable one to construct nontrivial "physical" examples of the general technique, discussed in this section, in subsequent work.

\section{ACKNOWLEDGEMENTS}

Research has been partially supported by EPSRC Grant GR/S13682/01. The linearizing substitution to equation (1.8) was pointed out by S. Yu. Sakovich. We would also like to thank the anonymous referee for carefully reading the paper and many valuable suggestions (which have been since incorporated herein). 


\section{REFERENCES}

[1] M. J. Ablowitz, H. Segur. Solitons and the inverse scattering transform. SIAM Studies in Applied Mathematics, 4. Society for Industrial and Applied Mathematics (SIAM), Philadelphia, $P A, 1981 . \mathrm{x}+425$ pp. MR0642018 (84a:35251)

[2] M. Boiti, F. Pempinelli, A. K. Pogrebkov, B. Prinari. Extended resolvent and inverse scattering with an application to KPI. Integrability, topological solitons and beyond. J. Math. Phys. 44 (2003), no. 8, 3309-3340. MR2006753(2004j:37137)

[3] J. M. Burgers. The nonlinear diffusion equation. D. Reidel, Massachusetts, 1974.

[4] C. Haselwandter, D. D. Vvedensky. Fluctuations in the lattice gas for Burgers' equation. J. Phys. A 35 (2002), no. 41, L579-L584. MR1946960 (2003k:82078)

[5] U. Frisch, J. Bec. "Burgulence". Turbulence: nouveaux aspects/New trends in turbulence (Les Houches, 2000), 341-383, EDP Sci., Les Ulis, 2001. MR1999766 (2004g:76075)

[6] J. D. Cole. On a quasi-linear parabolic equation occurring in aerodynamics. Quart. Appl. Math. 9 (1951) 225-236. MR0042889(13:178c)

[7] M. M. Crum. Associated Sturm-Liouville systems. Quart. J. Math. Oxford Ser. (2) 6 (1955) 121-127. MR0072332 (17:266g)

[8] F. Gesztesy, H. Holden. The Cole-Hopf and Miura transformations revisited. Mathematical physics and stochastic analysis (Lisbon, 1998), 198-214, World Sci. Publishing, River Edge, NJ, 2000. MR 1893107 (2003a:37107)

[9] E. Hopf. The partial differential equation $u_{t}+u u_{x}=u_{x x}$. Comm. Pure Appl. Math. 3 (1950) 201-230. MR0047234 (13:846c)

[10] S. B. Leble, M. A. Salle, A. V. Yurov. Darboux transforms for Davey-Stewartson equations and solitons in multidimensions. Inverse problems 4 (1992) 207-218. MR 1158176 (93h:35191)

[11] V. B. Matveev, M. A. Salle. Darboux Transformation and Solitons. Springer Verlag, BerlinHeidelberg 1991. MR:1146435 (93d:35136)

[12] A. C. Newell. Solitons in mathematics and physics. CBMS-NSF Regional Conference Series in Applied Mathematics, 48. Society for Industrial and Applied Mathematics (SIAM), Philadelphia, PA, 1985. xvi+244 pp. MR0847245 (87h:35314)

[13] P. J. E. Peebles. Principles of Physical Cosmology. Princeton University Press, 1993. MR $1216520(94 \mathrm{~d}: 83076)$

[14] S. Yu. Sakovich. On the Thomas equation. J. Phys. A 21 (1988) L1123-L1126. MR0980551 (90a:35209)

[15] A. V. Yurov. BLP dissipative structures in the plane. Phys. Letters A 262 (1999) no. 6, 445-452. MR1729501 (2000i:37131)

Department of Mathematics, University of Bristol, Bristol BS8 1TW, United KingDOM

E-mail address: m.rudnev@bris.ac.uk

Department of Theoretical Physics, Kaliningrad State University, Aleksandra Nevskogo 14, Kaliningrad 236041, Russia

E-mail address: artyom_yurov@mail.ru

Department of Mathematics, University of Missouri, Columbia, Missouri 65211

E-mail address: valerian@math.missouri.edu 